\title{
Experimental characterization of the distribution of collagen fiber recruitment
}

\author{
Sylvain Roy $\cdot$ Christophe Boss $\cdot$ Rana Rezakhaniha $\cdot$ \\ Nikos Stergiopulos
}

Received: 23 September 2010/Accepted: 14 February 2011/Published online: 26 March 2011

(C) Japanese Society of Biorheology 2011

\begin{abstract}
The passive biomechanical behavior of blood vessels is generally modeled by a parallel arrangement of elastin and collagen, with collagen recruitment depending on vessel strain. We experimentally determined the collagen recruitment distribution using confocal microscopy. Digital images from sections of rabbit carotid artery under increasing circumferential stretch ratio were acquired. The straightness of the fibers was measured to compute the fraction of recruited fibers for each stretch ratio. The experimental distribution obtained was then used in the model proposed by Zulliger et al. This model is based on a strain energy function (SEF), which provides a constituentbased description of the wall mechanics. Using this model, a fit of the pressure-radius curve was then performed by using the experimental collagen recruitment distribution with the collagen elastic constant as a free fit parameter. The fit was good, but the value of the collagen elastic constant obtained was below values reported in the literature. A second fit of the pressure-radius curve was also performed, whereby the collagen distribution was left free to adapt while the collagen elastic constant was set to a physiological value. The differences between the experimental collagen engagement distribution and the distribution obtained when the collagen elastic constant was fixed were analyzed. Good qualitative agreement was found between the experimental distribution of collagen recruitment and the model. Some experimental limitations and
\end{abstract}

S. Roy and C. Boss contributed equally to this work.

S. Roy $(\square) \cdot$ C. Boss $\cdot$ R. Rezakhaniha $\cdot$ N. Stergiopulos Laboratory of Hemodynamics and Cardiovascular Technology, Institute of Bioengineering, Swiss Federal Institute of Technology Lausanne (EPFL), Station 17,

1015 Lausanne, Switzerland

e-mail: sylvain.roy@epfl.ch modeling approximations remain. Nevertheless, experimental proof of progressive collagen recruitment was established, which validates the basic hypothesis of the model.

Keywords Collagen fiber - Arterial wall . Strain energy function

\section{Introduction}

SEF is the preferred method for describing the material proprieties of arteries. In recent years, major efforts have been put into developing structure-based SEFs, where parameters defining the SEF reflect the properties of the wall constituents and their structural interaction. An ideal SEF should be based on histological analysis to provide a better description of wall deformation under load.

The arterial wall is composed of three distinct elements: the vascular smooth muscles (VSM) that form the cellular part of the vessel, and the extracellular matrix major components elastin and collagen. Elastin is composed of multiple fibers grouped into sheets, forming several layers organized in the media; it is characterized by low elastic modulus $(\sim 0.4 \mathrm{MPa}$ [1]) that provides the compliance required to buffer the variation in blood pressure during the heart cycle. Elastin also bears most of the load at low pressures $(0-70 \mathrm{mmHg})$. Collagen fibers result from the arrangement of elementary units called procollagen fibers that are enzymatically processed to form larger bundles. The mechanical proprieties of collagen are characterized by high elastic modulus $(\sim 100-1000 \mathrm{MPa}$ [1]), making this material a very stiff structure. Collagen is distributed in the media in between the elastin laminae and the VSM. When collagen is not submitted to stress, the fibers are wavy, but they are straightened upon artery inflation and progressively engage to bear the pressure load. 
Collagen engagement distributions are commonly used in structural modeling of the arterial wall to take into account progressive fiber recruitment. The aim of this study is to collect histological information on the arterial wall structure to characterize collagen engagement experimentally. Confocal images from sections of rabbit carotid artery were acquired, and the straightness of the fibers was measured to determine the fraction of recruited fibers at different levels of circumferential extension. The experimental distribution was then used in the SEF previously developed by Zulliger et al. [2] to fit the experimental pressure-radius curve. The agreement between the model and the experimental distribution of collagen engagement was then analyzed to assess the agreement between the direct histological assessment of the collagen recruitment function and the theoretical model.

\section{Materials and methods}

\section{Artery collection}

Left common carotid artery $(\sim 50 \mathrm{~mm})$ was harvested from 6 male White New Zealand rabbits $(3.5 \pm 0.2 \mathrm{~kg})$ from a local slaughterhouse. Before sectioning the arteries, the longitudinal length in situ $(l)$ was measured for subsequent assessment of the physiological longitudinal stretch $\left(\lambda_{z}\right)$. The arteries were then transported to the laboratory at $4^{\circ} \mathrm{C}$ in phosphate-buffered saline (PBS) and cleaned with microsurgery scissors under a microscope to remove any excessive adventitial tissue. Artery length under no longitudinal stretch $(L)$ was then measured for calculation of $\lambda_{z}[3]$.

\section{Biomechanical analysis}

\section{Biomechanical testing}

Arteries were kept bathing in $\mathrm{PBS}$ at $37^{\circ} \mathrm{C}$ for the entire testing. Arteries were mounted on cannulae connected to an inflation testing system and were set to a physiological longitudinal stretch $\lambda_{z}$ of about 1.5. Arteries were then submitted to 5-6 preconditioning cycles (inflations and deflations ranging from 0 to $140 \mathrm{mmHg}$ at a rate of about $2 \mathrm{mmHg} / \mathrm{s}$ ). Pressurization of the system was achieved using a syringe pump (sp201iw; World Precision Instruments, FL, USA) connected to the arterial segment. Inflation and deflation pressure-diameter cycles were repeated to ensure repeatability of the curve and to minimize viscous effects [4]. Inflation pressure was recorded using a blood pressure transducer (BLPR; World Precision Instruments). External diameter data were measured with a high-accuracy digital charge-coupled device (CCD) micrometer (model LS-7030; Keyence, Germany). Pressure and external diameter data were collected using a data acquisition card (DAQ 6285; National Instruments, Austin, TX, USA) and analyzed using a built-in interface (7.1 Labview; National Instruments). VSM were inactivated by means of $10^{-1} \mathrm{M}$ sodium nitroprusside treatment given for $15 \mathrm{~min}$ before mounting the arteries.

\section{Geometrical analysis}

A small ring $(\sim 3 \mathrm{~mm})$ was cut off from each arterial segment to measure the opening angle, wall thickness, and inner and outer diameters. The rings were photographed with a light microscope at $3 \times$ magnification (Axioplan2; Zeiss, Germany). Each ring was then cut open and left to rest for $20 \mathrm{~min}$ in a PBS dish. The cut open ring was photographed under the same conditions to measure the opening angle (Fig. 1). Using a calibrated slide ruler as a reference, measurements of the internal and external diameter, arterial wall thickness, and opening angle were obtained using Image J $1.37 \mathrm{v}$ software (National Institutes of Health, Bethesda, MD, USA).

Histological analysis

\section{Mechanical conditioning}

Each artery was stretched at the physiological longitudinal stretch $\left(\lambda_{z}\right)$, and markings were placed every $6 \mathrm{~mm}$ using

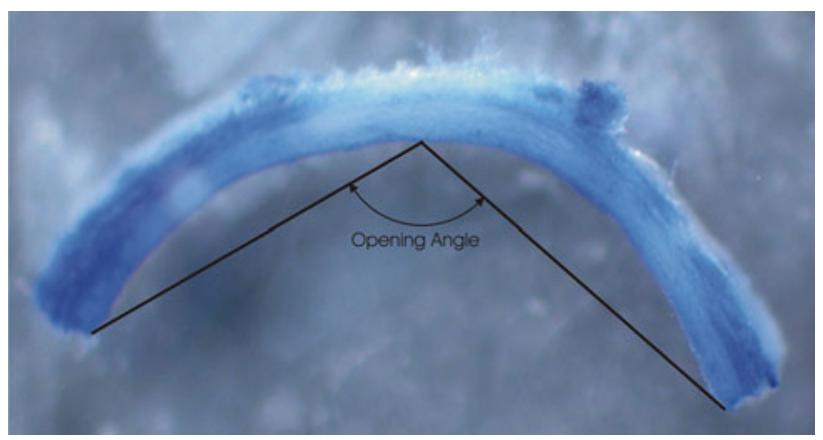

Fig. 1 Cut arterial ring photographed with a light microscope $(\times 3$ magnification) used to measure the opening angle

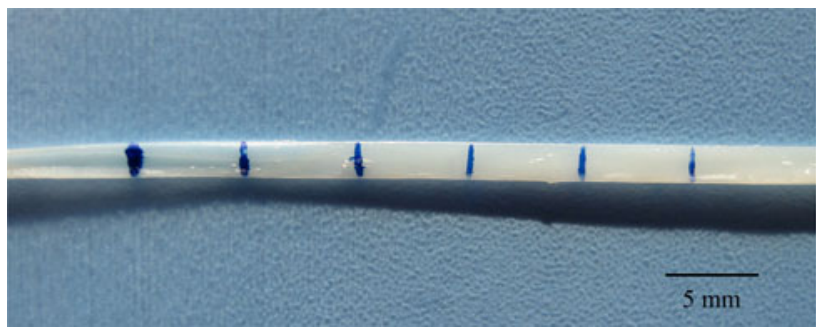

Fig. 2 Artery stretched at its physiological longitudinal stretch $\lambda_{z}$ marked every $6 \mathrm{~mm}$ using dark ink 
dark ink (Fig. 2). The artery was inserted onto a steel rod tapering at a slope of $2.5 \%$ to impose on the wall a progressive circumferential stretch varying along the $z$-axis. The longitudinal stretch was assumed to remain evenly distributed by checking the equal distance between each ink marking. The artery was then mounted in a chamber filled with polyvinyl alcohol (OCT Tissue-Tek; Sakura, Zoeterwoude, The Netherlands) and frozen at $-80^{\circ} \mathrm{C}$. The chamber was dismounted, and the rod withdrawn from the artery. The frozen artery, embedded in a conic polyvinyl alcohol cylinder, was mounted on a cryostat (Microm HM 560; Microm Intl, Walldorf, Germany). Five-micron-thick sections were cut to obtain a circumferential stretch $\lambda_{\theta}$ between 1.0 and 2.0 in steps of 0.1 . Sections were stained with Picro Sirius Red $0.05 \%$ to enhance collagen fiber autofluorescence [5].

\section{Confocal microscopy of collagen fibers}

Confocal images were acquired on a Leica TCS SP2 AOBS microscope. Collagen was visualized in the red spectra using an argon laser at $514 \mathrm{~nm}$ for excitation and a 525-600 nm bandwidth for emission. Elastin fiber autofluorescence was visible at $488 \mathrm{~nm}$. Sections were observed using a HCX PL APO $63 \times / 1.3$ immersion objective under $2 \times$ digital magnification (Leica, Wetzlar, Germany). Images were processed using ImageJ $1.37 \mathrm{v}$ software.

\section{Image analysis}

Collagen fibers present a wavy aspect that changes with the degree of circumferential stress. The reduction in such waviness, as a result of increased stress, can be expressed as the straightness of the collagen $(S)$, defined as the ratio of the length of the wavy fiber (Length wave ) to the length of the chord (Length chord ) connecting the two fiber ends (Fig. 3).

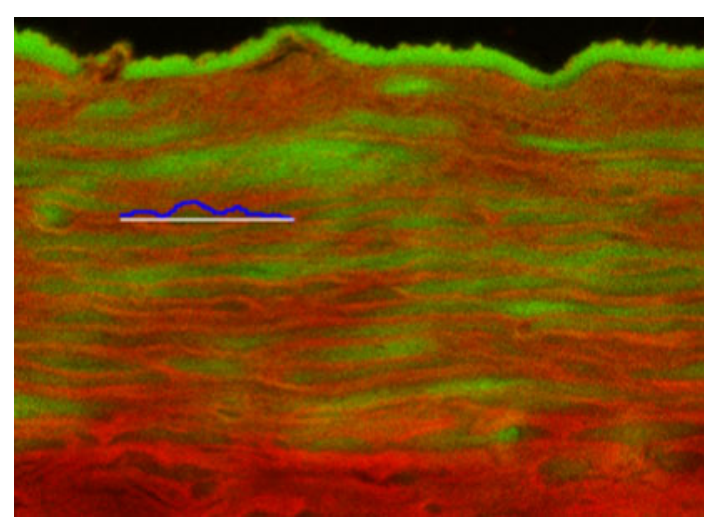

Fig. 3 Illustration of the straightness measurements. The length of the wave is given by the black line, and the length of the chord by the white line
$S=\frac{\text { Length }_{\text {chord }}}{\text { Length }_{\text {wave }}}$.

Measurements of the lengths were made on collagen fibers for each circumferential stretch $\lambda_{\theta}$ from 1.0 to 2.0 in 0.2 increments. For each of the 6 arteries, 40 measurements were performed per circumferential stretch. From these measurements a histogram showing the distribution of straightness was drawn for each $\lambda_{\theta}$ value. Straightness $\geq 0.998$ implies that the fibers were recruited to bear load. The log-logistic distribution $\mathrm{P}\left(\lambda_{\theta}\right)$ was used to fit the experimental distribution of collagen engagement by the least-squares method [2].

\section{Mathematical model}

\section{Three-dimensional description of the arterial wall}

The artery is considered to be a thick-walled tube made of nonlinear elastic incompressible material. We use a cylindrical coordinate system in which the stretch ratios are given by

$\lambda_{\theta}=\left(\frac{\pi}{\pi-\Theta}\right) \frac{r}{R}, \quad \lambda_{z}=\frac{l}{L}, \quad \lambda_{r}=\frac{\partial r}{\partial R}$

where $\Theta$ is the opening angle, $R$ is the radius to a point in the zero-stress state, and $r$ is the radius to the same point in the axially loaded, inflated state. $L$ is the axial length of the segment in the zero-stress state, and $l$ is the loaded axial length. Incompressibility is given by

$\lambda_{\theta} \lambda_{z} \lambda_{r}=1$

Inserting (2) into (3) and solving the resulting differential equation, we obtain

$r=\sqrt{r_{0}^{2}-\frac{(\pi-\Theta) L}{\pi l}\left(R_{0}^{2}-R^{2}\right)}$,

where $R_{0}$ is the outer radius in the zero-stress state and $r_{0}$ is the outer radius in the loaded, inflated state.

The Green strains are expressed in terms of the stretch ratios as

$E_{k}=\frac{1}{2}\left(\lambda_{k}^{2}-1\right) \quad k=\theta, r, z$

The local radial, circumferential, and longitudinal stresses in the arterial wall are given by

$\sigma_{k}=-p+\lambda_{k}^{2} \frac{\partial \Psi}{\partial E_{k}} \quad k=\theta, r, z$

where $\psi\left(E_{r}, E_{\theta}, E_{z}\right)$ is the SEF, which completely describes the mechanical properties of the wall material, and $p$ is the local hydrostatic pressure. Integrating the local stresses across the entire wall, we obtain the lumen pressure as 
$P=\int_{r_{\mathrm{i}}}^{r_{0}}\left(\sigma_{\theta}-\sigma_{r}\right) \frac{1}{r} \mathrm{~d} r$

where $r_{\mathrm{i}}$ is the inner radius and $r_{0}$ is the outer radius in the loaded state.

\section{Strain energy function}

We selected the SEF proposed by Zulliger et al. [2], which provides a constituent-based description of the passive wall mechanics. We assumed that elastin and collagen act in parallel and that their contribution to the total SEF is additive. The contribution of smooth muscle is neglected, being an order of magnitude smaller than that of elastin [6, 7]. A schematic view of the parallel arrangement of collagen and elastin is shown in Fig. 4.

The SEF is separated into an isotropic and an orthotropic part, representing elastin and collagen, respectively

$\Psi=f_{\text {elast }} \Psi_{\text {elast }}+f_{\text {coll }} \Psi_{\text {coll }}$,

where $f_{\text {elast }}$ and $f_{\text {coll }}$ are the fractions of the total wall crosssection area occupied by elastin and collagen, respectively. In previous work [8] it was found that a good description for the SEF of elastin $\Psi_{\text {elast }}$ is

$\Psi_{\text {elast }}=c_{\text {elast }}\left(I_{1}-3\right)^{3 / 2}$,

where the constant $c_{\text {elast }}>0$ is a parameter describing the elastic properties of elastin, and $I_{1}=2 E_{\theta}+2 E_{z}+$ $2 E_{r}+3$ is the first invariant of the Cauchy-Green strain tensor [9].

Collagen fibers appear to be coiled and wavy in their unloaded state $[10,11]$. We assume that the engagement of collagen as the artery is inflated and stretched is distributed in some statistical manner [8, 12, 13]. The log-logistic probability density function $(\mathrm{PDF}), \rho_{\text {fiber }}$ has been chosen for the engagement strain $E$ :

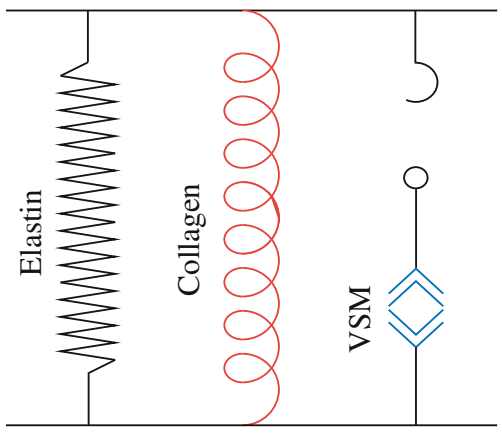

Fig. 4 Schematic representation of the parallel arrangement of collagen and elastin. Vascular smooth muscle is neglected in the passive state (low elastic modulus) $\rho_{\text {fiber }}(E)= \begin{cases}0 & \text { for } E \leq E_{0} \\ \frac{k}{b} \frac{\left(\frac{E-E_{0}}{b}\right)^{k-1}}{\left[1+\left(\frac{E-E_{0}}{b}\right)^{k}\right]^{2}} & \text { for } E>E_{0}\end{cases}$

where $b>0$ is a scaling parameter and $k>0$ is a parameter defining the shape of the distribution. The lower bound of the PDF is at a given value $E_{0}$, and we set $E_{0}=0$ to prevent any collagen from being loaded and thus exerting a force when the tissue is in its zero-stress state (ZSS). The fraction of fibers engaged at strain $E$ is given by

$P=\int_{-\infty}^{E} \rho_{\text {fiber }}(E) \mathrm{d} E= \begin{cases}0 & \text { for } E \leq E_{0} \\ 1-\frac{1}{\left(\frac{E-E_{0}}{b}\right)^{k}+1} & \text { for } E>E_{0}\end{cases}$

An individual collagen fiber is described by the SEF as

$\Psi_{\text {fiber }}\left(E^{\prime}\right)= \begin{cases}0 & \text { for } E^{\prime}<0 \\ \frac{c_{\text {coll }} E^{\prime 2}}{2} & \text { for } E^{\prime}>0\end{cases}$

where $c_{\text {coll }}$ is the elastic constant associated with the collagen and $E^{\prime}$ is the local strain in the direction of the fiber. To describe the SEF of all collagen fibers, we can convolute the SEF of a single fiber, $\Psi_{\text {fiber }}$, with the fiber engagement PDF, $\rho_{\text {fiber }}$, as

$$
\begin{aligned}
\Psi_{\text {coll }}(E) & =\Psi_{\text {fiber }} * \rho_{\text {fiber }} \\
& =\int_{-\infty}^{+\infty} \Psi_{\text {fiber }}\left(E^{\prime}\right) \cdot \rho_{\text {fiber }}\left(E-E^{\prime}\right) \mathrm{d} E^{\prime}
\end{aligned}
$$

We assume now that half the fibers are at an angle $\alpha$ to the circumference in the $\vec{e}_{\theta}-\vec{e}_{z}$ plane, which defines the surface of the cylinder at a specific $r$, and the other half at $-\alpha$ [3]. The stretch ratio $\lambda$ to which the fibers with $\alpha$ and $\alpha$ are submitted is the same and is given by

$\lambda=\sqrt{I_{4}}=\sqrt{\lambda_{\theta}^{2} \cos ^{2} \alpha+\lambda_{z}^{2} \sin ^{2} \alpha}$,

where $I_{4}$ is the fourth invariant of the Cauchy-Green strain tensor [9]. The total SEF (8), expressed according to the invariants $I_{1}$ and $I_{4}$, becomes

$$
\begin{gathered}
\Psi=f_{\text {elast }} c_{\text {elast }}\left(I_{1}-3\right)^{3 / 2}+f_{\text {coll }} \int_{-\infty}^{+\infty} \Psi_{\text {fiber }}\left(E^{\prime}\right) \\
\cdot \rho_{\text {fiber }}\left(\frac{I_{4}-1}{2}-E^{\prime}\right) \mathrm{d} E^{\prime}
\end{gathered}
$$

Model-fitting of the experimental pressure-radius curve

The computed pressure-radius curves were fitted to the experimental data by minimizing the residual sum of square $\Phi$ given by 
$\Phi=\frac{1}{n} \sum_{i}^{n}\left(r_{i}^{\text {model }}-r_{i}^{\text {experimental }}\right)^{2}$

where $i$ is the index of the acquired data and $n$ is the total number of data.

The opening angle, the inner and outer diameter, the fraction of collagen $f_{\text {coll }}$ and elastin $f_{\text {elast }}$, and the collagen angle $\alpha$ were experimentally determined and set in the model. The first fit was performed using the experimental PDF of the collagen engagement, letting the elastic constants of collagen and elastin adjust. The other fits were performed by setting the collagen elastic constant and by letting the PDF adapt. All computations were done using MatLab 7.3.0 (MathWork Inc., USA).

\section{Statistics}

Mean and standard errors are given for the diameter, the fraction of collagen and elastin, the straightness, and the fraction of recruited collagen fibers. The correlation of the collagen engagement distribution was determined as the correlation coefficient $r^{2}$. The parameters $k$ and $b$ for the collagen engagement distribution are given with a $95 \%$ confidence interval. The fits for the pressure-diameter curves were compared using the residual sum of squares $\Phi$.

\section{Results}

Histology

Confocal images of rabbit common carotid artery under progressive circumferential stretch $\lambda_{\theta}$ are presented in Fig. 5. Collagen fibers are visible as wavy structures that progressively straighten with increasing circumferential stretch. At low stretch, the fibers are relatively curly
Fig. 5 Images of arterial wall taken by confocal microscopy for different stretch ratios: a $\lambda_{\theta}=1.0, \mathbf{b} \lambda_{\theta}=1.2$, c $\lambda_{\theta}=1.4, \mathbf{d} \lambda_{\theta}=1.6$ e $\lambda_{\theta}=1.8$, and $\mathbf{f} \lambda_{\theta}=2.0$. Collagen is in red and elastin is in green (in the printed version, red appears dark gray and green appears light gray)
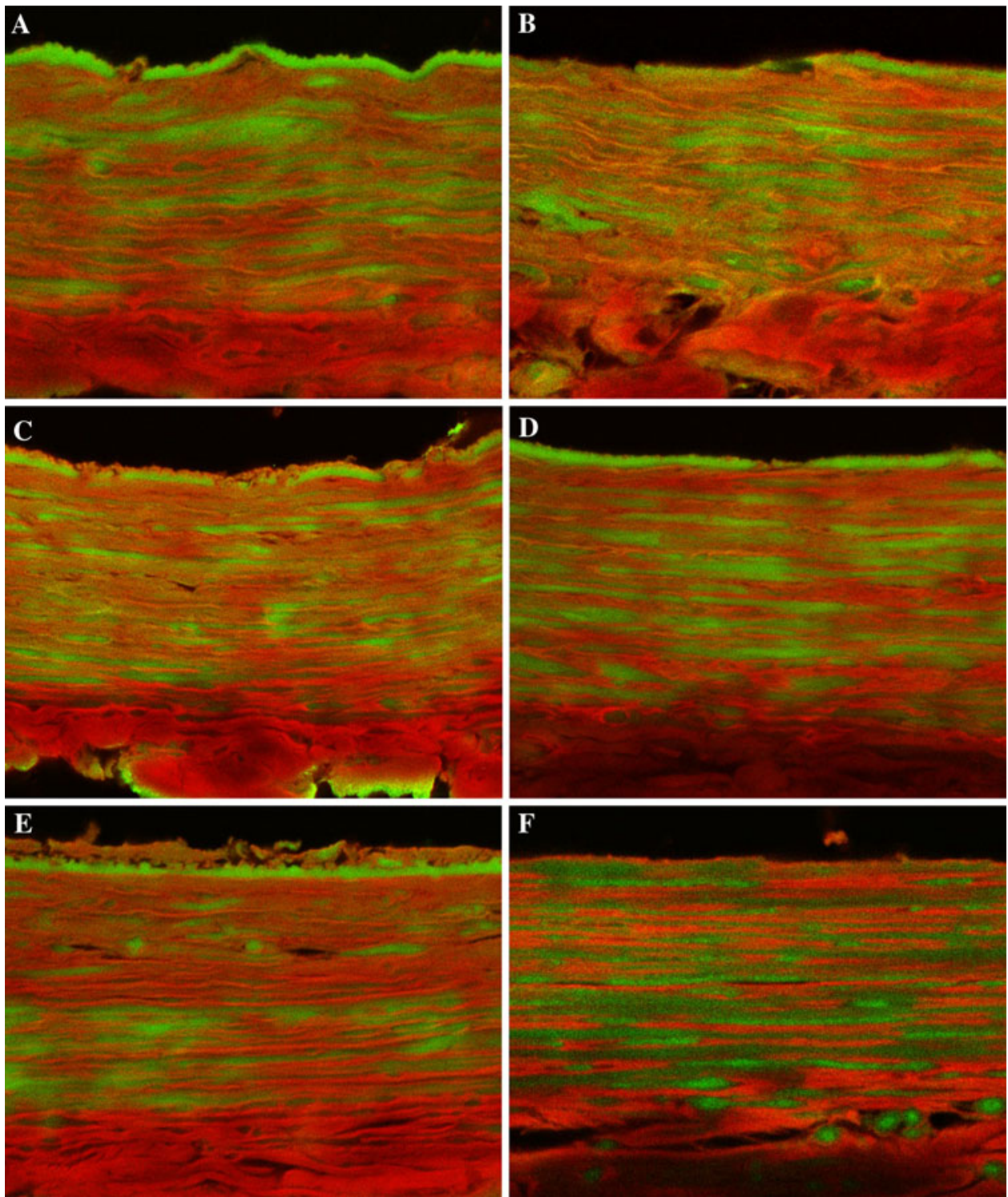
(Fig. 5a), and they become less and less undulating for $\lambda_{\theta}$ higher than 1.4 (Fig. 5c-f). Elastin appears as laminae running parallel to the collagen fibers.

Histograms of fiber straightness were obtained for increasing stretch ratios (Fig. 6). The straightness for $\lambda_{\theta}=1.0$ was $0.964 \pm 0.016$ (mean \pm standard deviation, SD), $0.988 \pm 0.010$ for $\lambda_{\theta}=1.6$, and reached $0.995 \pm 0.003$ for $\lambda_{\theta}=2.0$ (Fig. 6a, d, f). The distribution shifts to the right and becomes narrower with increasing stretch ratio, clearly indicating progressive straightening of the collagen fibers.

The experimental distribution of recruited fibers obtained from Fig. 6 and the fitted log-logistic distribution are presented in Fig. 7a. At $\lambda_{\theta}=1.7$, a stretch value corresponding to mean physiological pressure of $9.3 \mathrm{kPa}, 7 \%$ of the fibers are recruited. At $\lambda_{\theta}=2.0$, which is beyond the maximal physiological level of stretch, this value reaches
Fig. 6 Distribution of straightness measured on six arteries for increasing stretch ratio: $\mathbf{a} \lambda_{\theta}=1.0, \mathbf{b} \lambda_{\theta}=1.2$, c $\lambda_{\theta}=1.4$, d $\lambda_{\theta}=1.6$ e $\lambda_{\theta}=1.8$, and $\mathbf{f} \lambda_{\theta}=2.0$. With increasing stretch ratio, the distribution shifts to the right and becomes narrower and higher
Fig. 7 Experimental distributions of fiber recruitment according to the circumferential stretch $\lambda_{\theta}$ (a) and the Green strain $E$ (b) fitted with the loglogistic distribution (11). Most fibers are recruited between $\lambda_{\theta}=1.6$ and 2.0 , with $31 \%$ of fibers engaged at $\lambda_{\theta}=2.0$ (error bar $=\mathrm{SD})$
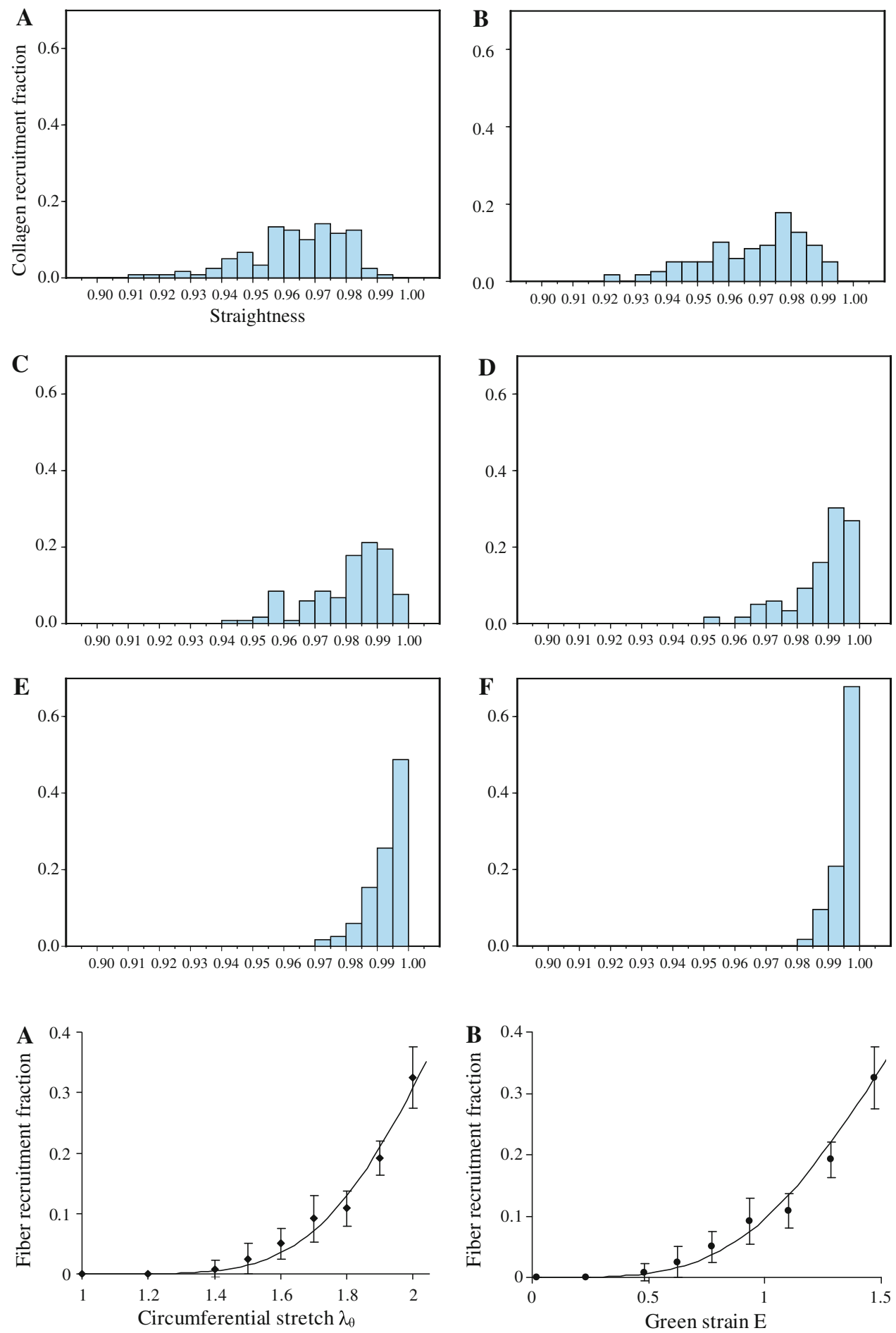
$31 \%$. Figure $7 \mathrm{~b}$ shows the fitted experimental distribution as a function of Green strain $E$. The fitted curve is characterized by $k=3.87$ [CI 3.07, 4.66] and $b=1.81$ [CI $1.68,1.94]$, the numbers in brackets giving the $95 \%$ confidence intervals. The correlation coefficient of the fit is $r^{2}=0.983$.

\section{Biomechanical analysis}

The zero-stress state parameters are the following: mean opening angle: $118.9 \pm 17.7$ degrees; inner and outer diameters: $1.45 \pm 0.08 \mathrm{~mm}$ and $1.99 \pm 0.12 \mathrm{~mm}$, respectively; mean wall thickness: $0.27 \pm 0.06 \mathrm{~mm}$. From histology, the collagen fraction was estimated as $f_{\text {coll }}=$ $0.43 \pm 0.04$ and the elastin fraction as $f_{\text {elast }}=0.27 \pm 0.03$.

Figure 8 shows the mean pressure-radius curve obtained experimentally. From 0 to $8 \mathrm{kPa}$, in the elastin-dominant domain, the curve is rather straight and steep. Above $10 \mathrm{kPa}$ the collagen engagement limits further diameter expansion and flattens the curve.

Figure 9a presents the fit between the experimental and model-predicted pressure-radius curves with parameters $k$ and $b$ from the collagen engagement PDF set to values found experimentally, and $c_{\text {coll }}$ and $c_{\text {elast }}$, the elastic constants for collagen and elastin, left free to vary. The result was $c_{\text {elast }} \approx 39.5 \mathrm{kPa}$ and $c_{\text {coll }} \approx 35.5 \mathrm{MPa}$ with a residual sum of squares of $\Phi=3.13 \times 10^{-4} \mathrm{~mm}^{2}$. The first part of the curve, until about $8 \mathrm{kPa}$, representing the elastin-dominated domain, is fitted very well, while the last part of the curve above $12 \mathrm{kPa}$ is less well fitted. The collagen engages earlier and is not stiff enough to flatten the curve sufficiently in the collagen-dominated domain.

The curve in Fig. 9b was obtained with the collagen elastic constant set at $500 \mathrm{MPa}$, a value chosen according to the literature [1], while $c_{\text {elast }}, k$, and $b$ were left free to vary for the best fit. The result was $c_{\text {elast }} \approx 41.0 \mathrm{kPa}$, $k=8.03$, and $b=1.43$ with a residual sum of squares of $\Phi=1.99 \times 10^{-4} \mathrm{~mm}^{2}$. As for Fig. 9a, the first part of the curve fits well but the last part remains not flat enough. The fit is, however, significantly better, the residual sum of squares being $35 \%$ smaller.

Figure 10a shows the distribution for the experimental collagen recruitment (continuous line) and the distribution obtained from the model with $c_{\text {coll }}=500 \mathrm{MPa}$ (dotted line) as a function of Green strain $E$. Figure 10 b shows the corresponding collagen recruitment PDF. In the
Fig. 8 Experimental pressureradius curves of six different arteries (a) and the corresponding mean curve (b)
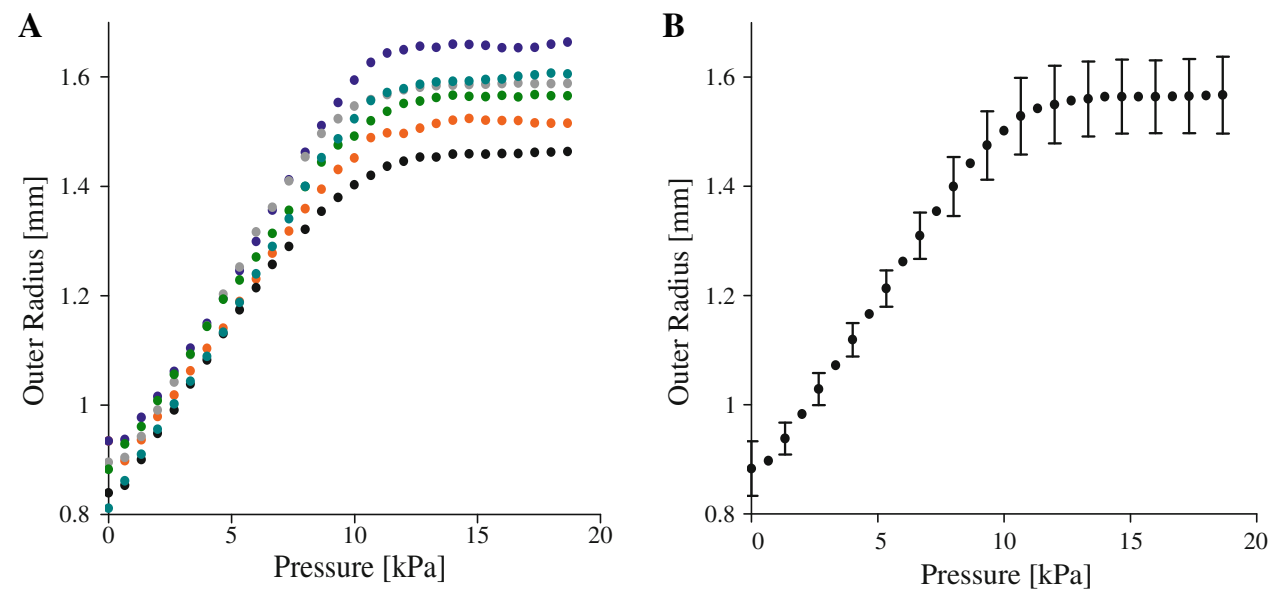

Fig. 9 Experimental mean pressure-radius curve fitted with the model. a Parameters $k$ and $b$ of the collagen engagement PDF were set to their experimental values, and $c_{\text {coll }}$ and $c_{\text {elast }}$, the elastic constants of collagen and elastin, were left free. $\mathbf{b} c_{\text {coll }}$ was set at $500 \mathrm{MPa}$ and $c_{\text {elast }}, k$, and $b$ were left free

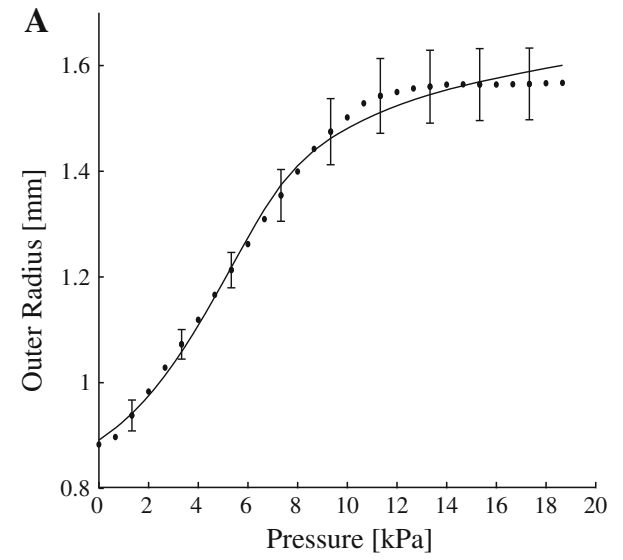


experimental distribution the collagen engagement begins early at strain of 0.4 , but increases slowly to reach about $30 \%$ at strain of 1.5 . In the model-based distribution, the collagen engagement starts later, from strain around 0.7, and increases quickly to reach about $60 \%$ at strain of 1.5 .

\section{Discussion}

The goal of this study is to experimentally determine collagen recruitment as a function of wall strain (PDF), which is used in constituent-based models of the arterial wall. Rather than indirectly deriving this collagen recruitment function by fitting the constituent-based model to pressurediameter data, we attempted to obtain it directly from experimental histological and morphological changes in the collagen structure upon inflation. We were also interested in analyzing the model of Zulliger et al. [2] by using experimental parameters instead of resorting to model-fit parameters. The results from the experimental and modelfit parameters are compared to bolster further refinements of the model.

The constituent-based SEF of Zulliger et al. [2] was selected for its efficiency in providing a good structural and 3-dimensional description of the arterial wall biomechanics. This function takes into account the nonlinearity of elastin and the anisotropic properties and wavy configuration of collagen. In the Zulliger model, the arterial wall is considered in a passive state (no smooth muscle tone) with all major structural components being organized in a parallel arrangement. The contribution of smooth muscle in its passive state to the total SEF and to the collagen configuration changes was considered negligible, the elastic constants of passive smooth muscle being an order of magnitude smaller than those of elastin [6, 7]. The Zulliger model has been repeatedly used in various modeling studies of the vascular wall and has been extended to include vascular smooth muscle [14], elastin anisotropy [15], and a differential zero-stress state and elastin content between the in-series and in-parallel elastic components [16]. The fact that the Zulliger model is a constituent-based model has allowed the study of the effects of different physiological or pathological effects on wall structure, such as the assessment of aging on elastic functionality of cerebral arteries [17], the effects of aging on the elasticity and collagen engagement properties of the human aorta [18], as well as the effects of hypertension on wall remodeling [19].

We have proposed a method to observe via confocal images the evolution of fiber straightness with increasing stretch ratio in order to derive the collagen fiber recruitment. It appeared to be difficult to distinguish directly, based on confocal images, recruited and nonrecruited fibers. Indeed, the waviness becomes very small with increasing stretch ratio, and fiber identification is difficult due to the mesh-like bundle organization of collagen. Furthermore, an arbitrary threshold for straightness to define the transition from a nonengaged to an engaged collagen fiber had to be implemented. Despite the above weaknesses and experimental drawbacks, an experimental distribution of collagen recruitment was derived and was expressed as the fraction of collagen fibers recruited per level of applied circumferential stretch (Fig. 7a). A 7\% recruitment at a stretch value $\lambda_{\theta}=1.7$ (corresponding to the mean physiological pressure) is in agreement with previous results. Bank et al. [20] found that $5-6 \%$ of collagen fibers were recruited at arterial pressure of $100 \mathrm{mmHg}$ in the in vivo human brachial artery, and Cox [21] calculated $10 \%$ collagen recruitment at $100 \mathrm{mmHg}$ for intact cylindrical segments of muscular canine artery.

The experimental distribution begins early but increases slowly compared with the model-based distribution (Fig. 10a). The value of the collagen elastic constant we obtained after fitting the pressure-radius curve using the collagen recruitment PDF determined from the experiments was very low $\left(c_{\text {coll }}=35.5 \mathrm{MPa}\right)$ compared with data reported from the literature [1]. The consequence of using such a low $c_{\text {coll }}$ was that early recruitment of many
Fig. 10 Collagen recruitment fraction (a) and PDF (b). Comparison of the collagen recruitment experimentally obtained (straight line) and with the model with the collagen elastic constant set at $500 \mathrm{MPa}$ (dotted line)
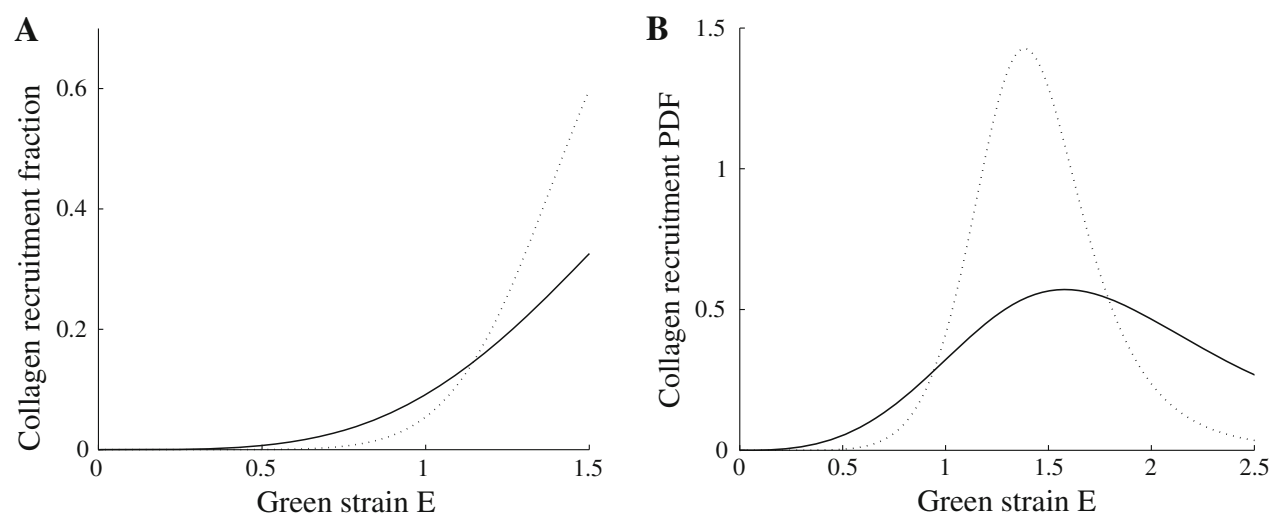
collagen fibers was necessary to compensate for the reduced stiffness of the individual fibers. Because the $c_{\text {coll }}$ was very low, the resulting tensile capacity of the collagen was limited, thus explaining the flatter PDF. Conversely, the model-based distribution engaged at higher stretch ratios, because $c_{\text {coll }}$ was one order of magnitude higher, implying that less collagen recruitment was required to achieve a comparable stiffening effect. The higher $c_{\text {coll }}$ resulted in stronger capability for collagen to withstand stress, thus limiting more efficiently the further increase in diameter at higher pressures and providing a flatter, more realistic pressure-radius curve at high pressures (compare Fig. $7 \mathrm{~b}$ and a). These observations suggest that stiff collagen engages later at a less rapid rate than soft collagen, because fewer fibers need to be recruited to reach the same stiffening result. The direct correlation between $c_{\text {coll }}$ and the PDF justified our choice to let $c_{\text {coll }}$ vary during the fit. The $c_{\text {coll }}$ being not known precisely, this procedure was an indirect manner to estimate $c_{\text {coll }}$. The estimated value (35.5 MPa), however, as stated above, lay below the range of values (100-1000 MPa) reported by Dobrin et al. [1], indicating that the experimentally derived PDF for collagen engagement was biased towards engagement at lower stretch ratios.

There are several limitations impacting on several methodological aspects of this study. Visualization of single collagen fibers is not evident with the confocal imaging technique employed. Scanning electron microscopy (SEM) images reveal much higher levels of waviness than seen in the confocal images. Furthermore, images were all taken on the transverse $(r-\theta)$ plane, whereas it is clear that most collagen fibers will be at an angle to the circumferential direction, and this angle will also follow some statistical distribution. The histograms of fiber straightness were not entirely smooth due to a limited number of measurements on the fibers (40/stretch/artery), which limits the precision of the fiber recruitment distribution. This statistical limit is partially responsible for the large standard deviations of the experimental distribution. As mentioned above, an important limitation also arises from the choice of the straightness criterion, which has an important impact on the shape of the collagen recruitment distribution.

In conclusion, we have determined an experimental distribution of collagen engagement using confocal images of rabbit carotid arteries. This distribution was used in a structural model of the arterial wall to compute a pressurediameter curve. This curve was fitted to pressure-diameter curves obtained from biomechanical testing on the same arteries by either letting the collagen recruitment distribution adapt or after setting the collagen elastic constant to a standard value. The collagen elastic constant obtained from the modeling was below usual values. Some differences were found between the experimental and the computed distribution of collagen engagement using a literaturederived collagen elastic constant. However, good agreement was found between the experimental distribution of collagen recruitment and the model. The general framework of the model appears to work well and reproduces plausible structural characteristics; however, further refinement of the imaging techniques for detailed visualization of individual collagen fibers is needed to achieve direct experimental validation of the model.

\section{References}

1. Dobrin PB. Mechanical properties of arteries. Physiol Rev. 1978;58:397-460.

2. Zulliger MA, Fridez P, Hayashi K, Stergiopulos N. A strain energy function for arteries accounting for wall composition and structure. J Biomech. 2004;37:989-1000.

3. Han HC, Fung YC. Longitudinal strain of canine and porcine aortas. J Biomech. 1995;28:637-41.

4. Fung YC. Biomechanics: mechanical properties of living tissue. New York: Springer-Verlag; 1993.

5. Montes GS. Structural biology of the fibres of the collagenous and elastic systems. Cell Biol Int. 1996;20:15-27.

6. Bergel DH. The static elastic properties of the arterial wall. J Physiol. 1961;156:445-57.

7. VanDijk AM, Wieringa PA, van der Meer M, Laird JD. Mechanics of resting isolated single vascular smooth muscle cells from bovine coronary artery. Am J Physiol. 1984;246:C277-87.

8. Wuyts FL, Vanhuyse VJ, Langewouters GJ, Decraemer WF, Raman ER, Buyle S. Elastic properties of human aortas in relation to age and atherosclerosis: a structural model. Phys Med Biol. 1995;40:1577-97.

9. Spencer AJM. Continuum mechanics. Essex: Longman Scientific \& Technical; 1980.

10. Clark JM, Glagov S. Transmural organization of the arterial media. The lamellar unit revisited. Arteriosclerosis. 1985;5: $19-34$.

11. Dingemans KP, Teeling P, Lagendijk JH, Becker AE. Extracellular matrix of the human aortic media: an ultrastructural histochemical and immunohistochemical study of the adult aortic media. Anat Rec. 2000;258:1-14.

12. Decraemer WF, Maes MA, Vanhuyse VJ. An elastic stress-strain relation for soft biological tissues based on a structural model. J Biomech. 1980;13:463-8.

13. Wolinsky H, Glagov S. Structural basis for the static mechanical properties of the aortic media. Circ Res. 1964;14:400-13.

14. Zulliger MA, Rachev A, Stergiopulos N. A constitutive formulation of arterial mechanics including vascular smooth muscle tone. Am J Physiol Heart Circ Physiol. 2004;287:H1335-43.

15. Rezakhaniha R, Stergiopulos N. A structural model of the venous wall considering elastin anisotropy. J Biomech Eng. 2008;130: 031017.

16. Roy S, Tsamis A, Prod'hom G, Stergiopulos N. On the in-series and in-parallel contribution of elastin assessed by a structurebased biomechanical model of the arterial wall. J Biomech. 2008;41:737-43.

17. Fonck E, Feigl G, Augsburger L, Rüfenacht D, Stergiopulos N. Biomechanical study of structural changes of human cerebral arteries. J Biomech. 2006;39:S364. 
18. Zulliger MA, Stergiopulos N. Structural strain energy function applied to the ageing of the human aorta. J Biomech. 2007;40:3061-9.

19. Tsamis A, Stergiopulos N. Arterial remodeling in response to hypertension using a constituent-based model. Am J Physiol Heart Circ Physiol. 2007;293:H3130-9.
20. Bank AJ, Wang H, Holte JE, Mullen K, Shammas R, Kubo SH. Contribution of collagen, elastin, and smooth muscle to in vivo human brachial artery wall stress and elastic modulus. Circulation. 1996;94:3263-70.

21. Cox RH. Regional variation of series elasticity in canine arterial smooth muscles. Am J Physiol. 1978;234:H542-51. 